\title{
Consideraciones psicológicas sobre el abordaje de la distonía focal en músicos
}

\author{
Psychological considerations on approaches to focal dystonia \\ in musicians
}

\author{
Karla María Reynoso-Vargas y Yander Amín Rodríguez-Simental ${ }^{1}$
}

\begin{abstract}
RESUMEN
En este texto se exponen tres consideraciones de abordaje clínico y metodológico en la distonía focal en músicos. La primera es observar los rasgos instrumentales y expresivos como factor que predispone a la patología; la segunda es considerar algunos aspectos socioculturales típicos del ámbito musical, tal como la competitividad, y la tercera es considerar los procesos psicoemocionales (ansiedad, duelo y adaptación) como detonadores al preparar un concierto.
\end{abstract}

Palabras clave: Distonía focal; Músicos; Psicología.

\begin{abstract}
The present paper describes three clinical and methodological considerations about focal dystonia in musicians. The first one consists of observing instrumental and expressive traits as a predisposing factor to this pathology. The second involves considering typical sociocultural aspects of musical environment, such as competitiveness. The third one considers psycho-emotional processes (anxiety, grief, and adaptation) as triggers, when they occur together during the preparation of an important concert.
\end{abstract}

Key words: Focal dystonia; Musicians; Psychology.

\section{INTRODUCCIÓN}

$\mathrm{L}$ as alteraciones músculo-esqueléticas originadas por la práctica musical son frecuentes. Se estima que hasta 95\% de los músicos de alto rendimiento desarrollará al menos una de tales alteraciones en algún momento de su carrera (Ackermann, Kenny, Fortune y Fortune, 2011). Algunas de estas afecciones son suficientemente importantes como para que afecten la ejecución musical y se agudicen a tal grado que puedan requerir la suspensión de la práctica.

Este ensayo se centra en la distonía focal, una patología considerada grave por los músicos pues frecuentemente termina con su carrera (Zosso y Schoeb, 2010). El objetivo de este trabajo es exponer algunas consideraciones psicológicas sobre ciertos aspectos que se dan por sentados al abordar este trastorno.

La distonía focal se define como un desorden de tareas específicas que se caracteriza por la descoordinación o pérdida del control motor voluntario al realizar movimientos en actividades como tocar un instrumento, que en la mayoría de los casos ocurre sin dolor, aunque ocasionalmente puede haber tensiones

\footnotetext{
${ }^{1}$ Escuela Superior de Música de la Universidad Juárez del Estado de Durango, Av. Nelly Campobello s/n, frente a la Secretaría de Recursos Naturales y Medio Ambiente, 34070 Durango, Dgo., México, tel. (618)130-11-21, correos electrónicos: kreynoso@ujed.mx y karmareynoso@gmail. com. Artículo recibido el 3 de octubre y aceptado el 11 de diciembre de 2017.

Citación: Reynoso-Vargas, K.M. y Rodríguez-Simental, Y.A. (2018). Consideraciones psicológicas sobre el abordaje de la distonía focal en músicos. Psicología y Salud, 28(2), 223-227.
} 
musculares ocasionadas por la compensación de los movimientos distónicos. Esta patología afecta aproximadamente a uno de cada cien concertistas (Ioannou y Altenmüller, 2014).

Se ha argumentado que la distonía focal y otras lesiones por sobreuso de las extremidades superiores -sobre todo los de tipo muscular-, son patologías con una base similar que evolucionan de forma distinta (Rosset-Llobet, 2015; Rosset-Llobet, Fábregas, Rosinés, Narbehaus y Montero, 2005).

La etiología de la distonía focal no ha sido comprendida por completo, aunque la evidencia sostiene que es multifactorial (Altenmüller y Jabusch, 2010; Altenmüller y Müller, 2013; Rosset-Llobet et al., 2005; Zosso y Schoeb, 2010). Su entendimiento debe considerar algunos aspectos psicológicos y técnicos musicales (Altenmüller y Müller, 2013), psicosociales (Altenmüller y Jabusch, 2010) y contextuales (Zosso y Schoeb, 2010).

Altenmüller y Jabusch (2010) han expuesto un modelo bastante aceptado en la literatura internacional que supone que la herencia genética y el género masculino son factores que predisponen al desarrollo de la distonía. El modelo indica que existen detonadores intrínsecos, tales como desórdenes físicos, lesiones por trauma y sobreuso, degradación de los circuitos sensoriomotores; así como detonadores extrínsecos, como las constricciones sensoriomotoras espacio-temporales y las restricciones sociales típicas de la situación del desempeño de la música clásica.

\section{Consideraciones psicológicas}

A continuación se exponen tres consideraciones psicológicas sobre ciertos aspectos que se dan por sentados en el abordaje de la distonía focal. La primera es que, según Rosset-Llobet et al. (2005), si bien el sexo masculino es el más afectado en una proporción de siete hombres por cada mujer, hasta ahora solo se ha explicado parcialmente la razón de tal diferencia. Altenmüller y Jabusch (2010) indican que el determinante en cuestión podría ser mas bien el género, es decir, la "fabricación cultural e histórica de lo que significa ser hombre o mujer" (Piñones, 2005). Dentro del género construido socialmente podría discurrirse sobre la interiorización de rasgos instrumentales, tales como el ser decidido, sistemático, responsable, diligen- te, cumplido, dedicado al trabajo y demás; así como rasgos expresivos, como ser comprensivo, afectuoso, generoso, conciliador, coorperador, dedicado a los demás y otros rasgos (Díaz-Loving, Rivera y Sánchez-Aragón, 2001), los cuales se asocian a los roles de género. Esta consideración deja de lado la idea de que dichas características sean exclusivas de únicamente uno de los sexos (Rocha y Ramírez, 2011) y permite explicar con más lógica la variación en la incidencia de la distonía.

Autores como Smiler y Kubotera (2010) han señalado que en el contexto laboral los hombres desarrollan más sus rasgos instrumentales y escasamente los expresivos. Tal tendencia puede vincularse a la discrepancia que hay entre el número de hombres y mujeres que sufren distonía. Los mismos Altenmüller y Jabusch (2010) encontraron indicios sobre cómo algunas cuestiones psicológicas se asocian con la distinta evolución en las patologías. En uno de sus estudios hallaron que, si bien había cierto traslape en las condiciones psicológicas, los distónicos manifestaban tendencias sumamente perfeccionistas y con tendencia a la fobia social; a diferencia de los músicos que desarrollaban dolor crónico, quienes resultaron ser más sensibles o emocionales que el grupo control, lo que coincide con la descripción de rasgos expresivos e instrumentales.

Una segunda consideración se refiere a que las aproximaciones a la distonía focal abordan superficialmente los aspectos socioculturales. Hay que discurrir que la enfermedad es un hecho sociológico, y que "cualquier acción de prevención, tratamiento o planeamiento de salud debería tomar en cuenta valores, actitudes y creencias de la población a la que está destinada" (Carbonell, 2006). Por lo tanto, habría que considerar los aspectos prevalentes en la comunidad de músicos, como la competitividad. En efecto, el campo musical es altamente competitivo desde la admisión a los programas superiores, hasta la selección de un limitado número de puestos laborales (Wristen, 2013). En este medio hay un marco subyacente de castigo y recompensa (Altenmüller y Jabusch, 2010) y una elevada tasa de perfeccionismo (Wristen, 2013). Aunque se ha sugerido que el perfeccionismo tiene un papel adaptativo (Rice, Leever, Chrisopher y Porter, 2006), también incide en el estrés que se deriva de los compañeros (Hunt y Eisenberg, 
2010). La actividad y el medio musical demandan un alto grado de competitividad, perfección, disciplina y exposición a la crítica de parte de quienes esperan tener éxito, y esas variables pueden ser mentalmente disruptivas (Powers, 2013; Wristen, 2013). Es posible que la competencia en el medio se asocie con el sobreuso y la ansiedad (Savino et al., 2013), factores que ya se han relacionado con el desarrollo de la distonía focal.

La tercera consideración está relacionada con la importancia de las emociones. Ya Altenmüller y Jabusch (2010) señalaron que la ansiedad es un factor detonante, pero la vincularon estrechamente con el perfeccionismo y las fobias, obviando la interacción con otros tipos de ansiedad, como la escénica (Ackermann et al., 2011; Powers, 2013), la relacionada con la depresión (Barbar, de Souza y de Lima, 2014; Hilderbrand, Nübling y Candia, 2012; Powers, 2013), con los intensos ritmos de trabajo musical (Savino et al., 2013) y la vinculada con otras áreas de la vida, a la ineficacia o inexistencia de estrategias adecuadas de afrontamiento (Hilderbrand et al., 2012) y a la competitividad del medio (Powers, 2013; Savino et al., 2013; Wristen, 2013); dejaron asimismo de lado el efecto de la depresión, ya asociada previamente al desarro1lo de lesiones (Powers, 2013; Wristen, 2013).

Debe recordarse que la cascada de catecolaminas y glucocorticoides provoca una desregulación del axis hipotálamo-pituitaria-adrenal. Los efectos excitatorios son reversibles cuando se trata de estrés aislado o poco intenso; pero si es repetido puede causar una atrofia en las dendritas de las neuronas del hipocampo (región CA3). De igual manera, el efecto del estrés crónico (mantenido durante meses o años) puede matar las neuronas. El hipocampo participa en la memoria verbal, la de contexto y la capacidad de evocación, y entraña los procesos de adquisición del aprendizaje y la memoria espacial.

El procesamiento de las emociones puede ser una pieza clave para comprender la etiología de la distonía del músico, sobre todo porque las estructuras cerebrales implicadas en el procesamiento de las emociones sobreponen el de la música a través del núcleo accumbens, amígdala, giro parahipocampal, córtex órbito-frontal, córtex temporal superior, córtex cingulado anterior, parte ventral del córtex premotor y regiones posteriores del giro inferoposterior, regiones que generalmente difieren en tamaño o en función en hombres y mujeres (Weisenbach et al., 2014).

Se sugiere especialmente tomar en cuenta los procesos psicoemocionales de duelo y adaptación que ocurren en conjunto antes de una presentación musical importante (recital o competencia), después de un periodo de tiempo en el que la persona se ha sentido estresada.

En el contexto clínico, los músicos relatan que antes de la aparición de la distonía focal preparaban un concierto que consideraban trascendental, a la par que sufrían una fuerte sensación de pérdida, que puede entenderse como estrés emocional derivado de la pérdida potencial o real de una relación significativa, que de alguna manera fue vinculada con la actividad musical.

Hay diversos ejemplos de lo anterior: un estudiante con síntomas de musicorexia que cortó el lazo de noviazgo en el periodo en que preparaba un recital relevante; un músico concertista reconocido internacionalmente, acostumbrado a viajar constantemente por la presentación de conciertos, que no pudo estar en el funeral de su hermano porque estaba de gira, o la enfermedad letal de una mascota muy querida durante la preparación de una audición escolar.

Otras formas de tal sensación de pérdida reportadas por los informantes fueron la pérdida de un cierto estilo de vida, el cambio de rutinas y del nivel de responsabilidad, y la pérdida de control sobre el ambiente, todos ellos con implicaciones en los procesos de adaptación psicológica y física.

La importancia de estos cambios adaptativos ha sido apuntada por otros referentes clínicos, tales como el Institut de l'Art Medicina y Fisiologia (2016), que ha indicado que "en casi todos los casos, entre las causas (de la distonía focal), se encuentra un cambio de rutina de trabajo en el contexto de un estrés psíquico más o menos importante (aumento de la intensidad, cambio de repertorio, preparación de conciertos, examen de oposición, etc.)".

Los aspectos antes mencionados se ilustran en el siguiente relato, citado en extenso, de un músico diagnosticado con distonía focal y rehabilitado después:

La distonía focal que padecí fue multifactorial. Estuve sometido a un ritmo de estudio intenso a lo largo de doce años continuos sin dejar de tocar [mi instrumento]. Comencé [a estudiar] a los 7 
años de edad y durante mucho tiempo fue un pasatiempo para mí. [A] los 16 decidí profesionalizar mi actividad y desde entonces tuve un ritmo lleno de estudio, conciertos, concursos, sin descanso alguno, incluidos los fines de semana. Al término de la licenciatura, decidí hacer una maestría en el extranjero en el mismo instrumento. Por cuestiones económicas, me vi forzado a terminar[la] en no más de dos años; esto me condujo a tener una rutina de estudio bastante exigente, sin descanso, y sesiones de recitales-exámenes rodeadas de mucha exigencia del medio, autoexigencia y estrés.

Dentro de los factores del desencadenamiento de la distonía estuvieron los cambios. En mi caso, cambié de instrumento. Pasé por un periodo de desadaptación a mi regreso a mi país de origen. Me encontré con el estrés y la depresión de no encontrar trabajo de inmediato. Cuando lo obtuve, el cambio en el ritmo de estudio al ritmo de trabajo fue drástico. Tuve varios conciertos importantes, entre ellos el de mi titulación de maestría frente a un jurado de nueve músicos de alto nivel y un público [constituido] principalmente por músicos; otro en mi ciudad natal, donde sentí un gran compromiso de no defraudar a familiares y amigos, e incluso a dependencias y autoridades que me habían apoyado para mis estudios. Todas estas circunstancias fueron en conjunto los factores que me predispusieron a padecer la enfermedad.

Tuve la suerte de estar enterado de qué era la distonía focal, lo que me permitió llegar a un diagnóstico a la brevedad y buscar solución [...]. Después de un largo proceso de rehabilitación neurológica, psicológica y psiquiátrica, pude llevar a un buen final mi enfermedad [...]. Me siento totalmente recuperado, y sin embargo padezco la pérdida de un ritmo de estudio intenso por el largo tratamiento de la rehabilitación. Actualmente me encuentro trabajando [para] recuperar la condición de antes.

El proceso de padecer distonía focal en mi mano derecha fue difícil; sin embargo, también me considero afortunado de haberla padecido, pues el aprendizaje a lo largo de la rehabilitación me hace sentir fortalecido y sobre todo vivo, lo que me permite disfrutar mi actividad cada se- gundo sin tanta presión, autoexigencia, perfección, estrés, etcétera.

Durante el proceso de recuperación tuve un aprendizaje muy importante en cuanto a la ansiedad escénica del músico que me ha permitido aplicarlo con mis alumnos y ayudarlos a mejorar. También he aplicado una variedad de ejercicios técnicos y de repertorio para evitar movimientos repetitivos. Los he hecho más conscientes de su cuerpo, postura y relajación, lo que les permitirá [evitar] caer en alguna posible lesión. Aun así, creo que el principal mensaje que les proporciono es el de que, a pesar de la adversidad, la tenacidad, la fuerza de voluntad y la constancia pueden vencer una enfermedad como la distonía focal. Nadie está exento de padecer alguna lesión. Mi labor ha sido tratar de entender que, por citar un ejemplo, al igual que un futbolista puede lesionarse en algún juego, no sale con miedo a la cancha; se enfrenta sabiendo que existe la posibilidad de lesionarse; sin embargo, es consciente y entrena, [se] prepara adecuadamente, y si eso llegase a suceder, lo tomaría como algo que es más común de lo que creemos, lo enfrenta, lo supera y vuelve a la cancha.

Como profesor, inicialmente fue difícil debido a que no podía tocar en clase y de alguna manera ejemplificar lo expuesto en esta. Sin embargo, eso [me] permitió el desarrollo de otras habilidades que, en general, todo músico debería realizar con o sin distonía: digitar visualmente, escuchar o ver y determinar cómo se puede mejorar un cierto gesto, la digitación, la dirección musical... Me permitió [también] aprender a ver las necesidades reales del alumno a partir de sus propias capacidades y no a partir de [las mías]. Invito a las personas que la padecen o la padecerán a tomarlo con mucha calma y verlo como una pausa en su carrera que les permitirá regresar con mucha más fuerza física y mental.

Se espera que las consideraciones presentadas antes contribuyan al abordaje clínico de la distonía focal, así como a planteamientos metodológicos para futuros estudios que discurran los factores psicológicos y socioculturales que pueden conducir a caracterizar el entramado causal de la distonía focal en los músicos. La prevención de dicha 
condición es importante no solo en términos de salud física, sino también porque las largas interrupciones en la ejecución a causa del tratamiento, generalmente, causan complicaciones psicológi- cas, el retroceso del nivel técnico musical y diversas limitaciones laborales y sociales de quien lo padece (McCready y Reid, 2007).

\section{REFERENCIAS}

Ackermann, B.J., Kenny, D.T., Fortune, K. y Fortune, J. (2011). Incidence of injury and attitudes to injury management in skilled flute players. Work, 40, 255-259. doi: 10.3233/WOR-2011-1227.

Altenmüller, E. y Jabusch, H.C. (2010). Focal hand dystonia in musicians: phenomenology, etiology, and psychological trigger factors. Journal of Hand Therapy, 22, 144-155.

Altenmüller, E. y Müller, D. (2013). A model of task-specific focal dystonia. Neural Networks, 48, 25-35.

Barbar, A.M., de Souza Crippa, J.A. y de Lima O., F. (2014). Performance anxiety in Brazilian musicians: Prevalence and association with psychopathology indicators. Journal of Affective Disorders, 381-6, 152-154. doi: 10.1016/j.jad.2013.09.041

Carbonell, B. (2006). Cultura y diversidad en salud (inuits, kariñas, mapuches, saharuies). Bariloche (Argentina): Universidad Fasta-S.C.

Díaz-Loving, R., Rivera A., S. y Sánchez-Aragón, R. (2001). Rasgos instrumentales (masculinos) y expresivos (femeninos), normativos (típicos e ideales) en México. Revista Latinoamericana de Psicología, 33(2), 133-139.

Hilderbrand, H. Nübling, M. y Candia, V. (2012). Increment of fatigue, depression, and stage fright during the first year of high-level education in music students. Medical Problems of Performing Artists, 27, 43-48.

Hunt, J.H. y Eisenberg, D. (2010). Mental health problems and help-seeking behavior among college students. Journal of Adolescent Health, 46, 3-10.

Institut de l'Art Medicina y Fisiologia (2016). Distonía focal del músico. Recuperado de http://www.institutart.com, (27 de enero de 2016).

Ioannou, C.I. y Altenmüller, E. (2009). Psychological characteristics in musician's dystonia: A new diagnostic classification. Neuropsychologia, 61, 80-88.

McCready, S. y Reid, D. (2007). The experience of occupational disruption among student musicians. Medical Problems of Performing Artists, 22(4), 140.

Piñones, P. (2005). La categoría de género como dispositivo analítico en la educación. Memoria del primer Foro Nacional "Género en Docencia, Investigación y Formación de docentes (p. 27). México: INMUJERES-PNUD.

Powers, K. (2013). Young musicians face depression with silence. Teaching Music, 21(1), 24.

Rice, K.G., Leever, B.A., Chrisopher, J. y Porter, J.D. (2006). Perfectionism, stress, and social (dis)connection: A short-term study of hopelessness, depression, and academic adjustment among honors students. Journal of Counseling Psychology, $53,524-534$.

Rocha S., T.E., y Ramírez G., R.M. (2011). Identidades de género bajo una perspectiva multifactorial: Elementos que delimitan la percepción de autoeficacia en hombres y mujeres. Acta de Investigación Psicológica, 1(3), 454-472.

Rosset-Llobet, J. (2015). El dolor en los músicos. Barcelona: Institut de l'Art Medicina y Fisiologia. Recuperado de http://www. institutart.com/index.php/es/divulgacio/item/dolor.

Rosset-Llobet, J., Fábregas, S., Rosinés, D., Narbehaus, B. y Montero, J. (2005). Análisis clínico de la distonía focal en los músicos. Revisión de 86 casos. Neurología, 20(3), 108-115.

Savino, E., Iannelli, S., Forcella, L., Narciso, L., Faraso, G., Bonifaci, G. y Sannolo, N. (2013). Muscoloskeletal disorders and occupational stress of violinists. Journal Of Biological Regulators And Homeostatic Agents, 27(3), 853-859.

Smiler, A.P. y Kubotera, N. (2010). Instrumental or expressive? Men \& Masculinities, 12(5), 565-574.

Weisenbach, S.L., Rapport, L.J., Briceno, E.M., Haase, B.D., Vederman, A.C., Bieliauskas, L.A. y Langenecker, S.A. (2014). Reduced emotion processing efficiency in healthy males relative to females. Social Cognitive \& Affective Neuroscience, 9(3), 316-325.

Wristen, B.G. (2013). Depression and anxiety in university music students. Applications of Research in Music Education, 31(2), 20-27.

Zosso, A. y Schoeb, V. (2012). Musicians' social representations of health and illness: A qualitative case study about focal dystonia. Work, 41, 53-59. 